\title{
Two Cases on Holistic Care of Older Adults
}

\author{
Lee Hyer, PhD, ABPP ${ }^{1}$, Kristin A.J.Wagner, M.S. ${ }^{2}$ \\ Mercer School of Medicine and Georgia Neurosurgical Institute \\ lhyer@ganeuroandspine.com
}

\begin{abstract}
Holistic training involves a variety of cognitive behavioral techniques designed to improve overall health as well as delay the onset of neurodegenerative diseases such as those associated with aging. This is a multifaceted approach, incorporating Cognitive Training (CT), nutrition, physical activity, socialization, stress reduction, and general health.While the research on CT is not conclusive, it has been shown to have clinical utility, particularly with motivated subjects who have some internal and external resources. Two such cases are discussed. These subjects, both older adults, participated in a holistic Memory Clinic. Each had particular weaknesses and strengths, and both showed some improvement.
\end{abstract}

Joe is a $69 \mathrm{y} / \mathrm{o}$ male referred for an evaluation for cognitive problems and depression. He was an accountant who had been very successful. He retired in 2009 and has been living in Dallas until he had an accident in 2015. He abused alcohol, fell, and had a concussive disorder. He has been in recovery/rehabilitation for many months and now is residing in an assisted living facility (ALF) with his sister-in-law as his POA. On testing, Joewas high average premorbidly, but now showed problems in memory, attention, and visuospatial areas. He is also depressed and anxious. He also has pervasive obsessions that freeze him from any decision. In sum, he has cognitive problems, is depressed, and is detached and awkward socially with OCD.

Mary, on the other hand, is a $76 \mathrm{y} / \mathrm{o}$ female also referred for an evaluation for memory problems. She had a long career as a secretary but now lives with her husband. She has had substantial memory problems for over two years. In fact, this has been in process for many years. She is impaired, cannot do all her activities of daily living, and is struggling. She receives support on medications, finances, and tasks that are complex. On testing and interview, she is experiencing a mild dementia. She has special problems in language (anomia), memory, new learning, and executive functioning. She is also mildly depressed.

Both cases reflect common problems of older adults, one more severe than the other. Recently, two consensus statements about brain training offered conflicting viewson the validity. One argued that no compellingevidence exists to support the claims of brain-trainingcompanies that brain games (Cognitive Training (CT)) enhance cognition or staveoff the cognitive consequences of aging (Stanford Group); the other noted extensive supportfor scientifically grounded brain-training interventions (John Hopkins Group). Reading between the lines, it was evident that strict science (adequate controls, far transfer effects, sample size, etc.) cannot support its validation, yet clinical application is probably warranted. Factually, most studies have reported relative benefits of cognitive training in regard to narrow transfer and are experienced as positive for completers. Additionally, subjects who are motivated, have some skills to start with, and have support, do well.

We have advocated for holistic training (Hyer, 2014). We had argued that CT especially makes sense in the context of a lifestyle change and supportive system. We conducted two studies, one on computer CT and one on a holistic lifestyle trainingemphasizing CT. In the former case, we examined the effectiveness of Cogmed, a computerized program that trains working memory. We assessed this program against a sham group in older adults who have Mild Cognitive Impairment (MCI). Older adults ( $=68$ ) living in the community were assessed. All participants reported and demonstrated memory impairment during 
initial screening, but presented with normal MMSEs, ADLs, and IADLs. They met criteria for MCI. A total of 25 sessions were completed over 5-7 weeks. Pre-, post- and follow- up measures included a battery of cognitive and functional measures.Both intervention groups improved over time. The Cogmed group, however, scored higher on follow-up scores of functioning and adjustment. The Cogmed group had many significant post and follow-up changes relative to baseline (greater than Sham). In addition both groups, but especially Cogmed, enjoyed the intervention (Hyer , 2014).

For the latter study, we conducted an evaluation involving a "holistic" training program for several cohorts of older adults $(\mathrm{N}=112)$, targeting community residents with a spectrum of memory complaints ranging from Age Associated Memory Impairment to mild dementia. We developed a 7-session, manualized program targeting concentration, as well as mindfulness, exercise, stress reduction, socialization, diet and coping techniques. We applied this model to 11 cohorts and conducted pre and post testing on memory, memory habits, as well as function. In addition, we had a Control Group who received no cognitive training. We also divided the Memory Group by Risk Status-Low, Medium and High. Results showed that the memory group improved on this training on virtually all scales. The Lowand Medium Risk Groups(less problems) were statistically superior to the other groups including Control on most cognitive measures. There were differences also on memory complaints and adjustment, again favorably for the Low and Medium Risk Groups. Identifying high and low risk subjects was most helpful, resulting in clear differences between memory complaining older adults(Hyer, Scott, Lyles, Dhabliwala, \& McKenzie, 2014).

We believe that a more holistic behavioral approach to improving brain performance -- a combination of $\mathrm{CT}$, diet, exercise, socialization, stress reduction and general health - delays the onset of neurodegenerative diseases, such as dementia. Other programs have been applied with success with more impaired older adults including dementia (e.g., Logsdon, 2010;Zarit, Femia, Kim, \&Whitlach, 2010)A growing body of research seems to show also that early support groups can be beneficial (e.g., Snyder, Jenkins, \& Johnson, 2007). Often the focus involves a caregiver and provides psychoeducation, behavioral activation, and monitoring of targeted problem areas. In addition, environmental enrichment (occupation, leisure, complexity in life, mental training) and a health focus, notably enhanced cardio-vascular health and neurocognitive function (Hertzog et al., 2009), and exercise (Kramer et al. 2009), are impactful. More demanding tasks are especially helpful, as there is increased activity in the brains' frontal regions. This protects cognitive function and may be strengthened by cognitive and social engagement as well as physical exercise (Park \& ReuterLorenz, 2009). Finally, engagement in intellectually and socially stimulating activities (e.g., reading books, puzzles, volunteer work, traveling, card games, etc.) has been shown to enhance thinking and memory, and attention control processes, thereby increasing brain reserve capacity (e.g., Carlson, et al., 2009).

The core technique in our Memory Clinic is memory itself, emphasizing attention and concentration. The method that is promoted during Memory Clinic involves atechnique entitled "WAARR" -- Wake up (e.g., move saccades), Ask (What do I know?), Act (do the task), Recall (look away and recall), and Repeat (do all over). Throughout the sessions the participants perform various mental exercises related to WAARR. This technique maximizes core memory features of brain activation (concentration), association (what do I know), active engagement, recall and repetition. They are requested to practice this hourly as a method to stave off autopilot activity. They have day-minders to encourage this. To foster this, the facilitator utilizes behavior modification techniques, such as self-monitoring, to promote more ownership of their memory. Again, we teach this in each session and enter the additional markers of exercise, stress reduction, mindfulness and relaxation, diet, socialization, and general health, one for each of the sessions.

In the two cases above, each person was assessed and provided a plan based on the CT and on the six spokes of the Memory Clinic. The cases differ by severity of their problems, nonetheless both are applicable for the model. Joehad the good fortune to be in anALF where there was support for CT and several other 
model markers. We coordinated the model with them. During the memory clinic itself, he continued to show difficulty with retrieval and attending to directions. Joe's problem solving skills and understanding of directions increased over time, however. Socializing was targeted and he partook in exercises during opening activities. He was able to communicate and interact skillfully with fellow participants. He went to movies with others and took a trip to the Biltmore Estate. His health was discussed and monitored in the primary care clinic where the training took place. He was also taught stress reduction methods (breathing and acceptance challenges for OCD). He noted, during our discussion of diet and nutrition that, although his diet is fairly controlled by the facility, it was fairly close to the Mediterranean diet. The clinic advocated exercise in the facility gym and he complied. Joe was cooperative with a yoga exercise and agreed to monitor and increase his everyday physical activity. Overall, Joe was cooperative and compliant on targeted exercises. His scores reflected improvement, he now has a driver's license, and he plans to move out on his own.

Mary is of course a different case. She quickly became a widow during the study as her husband who was sickly died and is now assisted by her son who has moved in. She is grieving. A careful plan was developed for her that included the assistance of her son and other family members. She can drive, do shopping, and handle basic home tasks of cleaning and cooking. She has help with her medications and finances as well as health (cancer survivor). She also has age-related hearing loss impacting her social functioning. Maryindicated that she is not comfortable wearing her hearing aids. That said, she was requested to use them for targeted tasks. She agreed. The overall plan involved the core CT exercises of attending the Memory Clinic, doing puzzles at home, and practicing WAARR. She was reluctantly compliant. Her son was her coach. She was to see the clinic physician every two months to assure health. She was overweight and with no exercise program. She agreed to take up bowling and walking. This was monitored. Her diet was now monitored by her son who was a chef. Now an added problem was her husband's death. She was depressed and anxious, frustrated with past reactions to her husband's drinking. She was now crying frequently. She was validated for this and given two strategies for crying, including isolating times for this. Cognitively it is noteworthy that she had substantial language problems and she communicated poorly with those around her. She was also provided strategies for this including an accepting position on her condition. She also gave herself time and wrote down statements or at least core words.

Additionally, her attention is poor and she has difficulty focusing, with her hearing again being a major hindrance. She has problems understanding and following directions. Her processing speed is low and she has trouble organizing and making use of information. Mary's recall is also poor on both short term and delayed tasks. She has trouble remembering her phone number and the day and time, for example. She was again instructed to use repetition and a white board for the day's activity. She of course practiced WAARR. During the memory clinic, Mary struggled to socialize. Increasingly she participated in team activities, with her hearing being helping. With practice, she could remember a series of words in a semantic category. She again had trouble attending to and following directions when asked. In the exercise portion of the clinic, Mary had difficulty completing the exercises, but she gave good effort. Always she was rewarded and accepted. She was unsure if she would be able to continue an exercise program or maintain a healthy diet at home. She did. Her son was very helpful in all these areas. Overall, her prognosis was slow but steady. She has shown no noticeable deficits in one year.

We believe that the optimal care for the various designations of Age Associated Memory Impairment, Mild Cognitive Impairment (now Mild Neurocognitive Disorder) or dementia is a structured care program that includes the Memory Clinic model, a holistic program. These two cases highlight this and suggest that a careful case management plan with targeted interventions, simple supports, and built-in monitoring and rewards can make a difference, even in older adults with a poor prognosis. 


\section{REFERENCES}

1. Carlson, MC et al. (2009). Evidence for neurocognitive plasticity in at-risk older adults: The experience corps program. The Journals of Gerontology. Series A, Biological Sciences and Medical Sciences, 64(12), 1275-1282.

2. Hertzog, C., Kramer, A.F., Wilson, R.S., \&Linderberger, U. (2009). Enrichment effects on adult cognitive development: Can the functional capacity of older adults be preserved and enhanced? Psychological Science in the Public Interest, 9, 1-65.

3. Hyer, L. (2014). Psychological Treatment of Older Adults: A Holistic Model. New York: Springer.

4. Hyer, L., Scott, C., Lyles, J., Dhabliwala, J., McKenzie, L. (2014). Memory intervention: The value of a clinical holistic program for older adults with memory impairment. Aging and Mental Health, 18(2), 169-178.

5. Kramer, A., Erickson, J. and Colcombe (2009).Exercise, cognition, and the brain.

6. Logsdon, R.G., Pike, K.C., McCurry, S.M., Hunter, P., Maher, J., Snyder, L., Teri, L. (2010). Early-stage memory loss support groups: Outcomes from a randomized controlled clinical trial. The Journals of Gerontology Series B: Psychological Sciences and Social Sciences, 65(6), 691-697.

7. Park, D.C., \& Reuter-Lorenz, P. (2009). The adaptive brain: Aging and neurocognitive scaffolding. Annual Review of Psychology, 60, 173-196.

8. Snyder, L., Jenkins, C., \&Joosten, L. (2007). Effectiveness of support groups for people with mild to moderate Alzheimer's disease: An evaluative survey. American Journal of Alzheimer's Disease and Other Dementias, 22(1), 14-19.

9. Zarit, S.H., Femia, E.E., Kim, K., \&Whitlach, C.J. (2010). The structure of risk factors and outcomes for family caregivers.

Citation: Lee Hyer, PhD, ABPP, Kristin A.J.Wagner, M.S., "Two Cases on Holistic Care of Older Adults". American Research Journal of Geriatrics and Aging; Volume 2017; pp:1-4

Copyright (c) 2017 Lee Hyer, PhD, ABPP, Kristin A.J.Wagner, M.S., This is an open access article distributed under the Creative Commons Attribution License, which permits unrestricted use, distribution, and reproduction in any medium, provided the original work is properly cited. 